\title{
Efecto del periodo seco en cabras bajo condiciones de pastoreo, en el volumen y cantidad de compuestos lácteos subsecuente
}

\author{
Effect of dry-off period in goats under grazing conditions, on milk production and compounds quantity
}

\begin{abstract}
Ricardo Avilés Ruízz,2, José Alberto Delgadillo Sánchez', José Alfredo Flores', Manuel de Jesús Flores Nájera3 ${ }^{3}$ Alexis Adrián Vargas Cruz', Oscar Barrón Bravo ${ }^{2}$, Andrés Sánchez Hernández', Horacio Hernández Hernández ${ }^{1 *}$

Centro de Investigación en Reproducción Caprina, Posgrado en Ciencias Agrarias, Universidad Autónoma Agraria Antonio Narro. Periférico Raúl López Sánchez. C.P. 27054. Torreón, Coahuila, México.

2 Instituto Nacional de Investigaciones Forestales, Agrícolas y Pecuarias, Campo Experimental Las Huastecas, Villa Cuauhtémoc carretera Tampico Mante kilómetro 55. C.P. 89610. Altamira, Tamaulipas, México.

3 Instituto Nacional de Investigaciones Forestales, Agrícolas y Pecuarias, Campo Experimental La Laguna, Matamoros, Boulevard José Santos Valdez. C.P. 27440. Matamoros, Coahuila, México.
\end{abstract}

\section{RESUMEN}

El objetivo del presente fue determinar si el practicar el periodo seco en cabras bajo condiciones de pastoreo incrementa la producción láctea y la cantidad de los componentes químicos de la leche. En el grupo de cabras sin periodo seco los animales se mantuvieron diariamente en ordeño manual hasta la ocurrencia del parto (SPS; $n=9$ ). En las cabras del grupo con periodo seco se practicó el secado al suspender el ordeño manual desde 50 días antes del parto (CPS; $\mathrm{n}=11)$. Se utilizó un diseño factorial con un modelo lineal de efectos fijos para evaluar la producción de leche y la cantidad de sus componentes en respuesta al tratamiento, el factor tiempo y la interacción tiempoxtratamiento. Existió un efecto del tratamiento sobre la producción de leche durante la lactancia, por lo que ésta fue mayor para el grupo CPS que para el grupo SPS $(P<0.001)$. De igual manera la cantidad de componentes de la leche mostró un efecto significativo del tratamiento $(P<0.001)$. Se concluye que en cabras lactantes mantenidas en condiciones de pastoreo el practicar el periodo seco promueve una mayor producción de leche en la subsiguiente lactancia y por ello también una mayor cantidad de sus contenidos.

Palabras clave: Periodo seco, Cabras, Lactancia, producción de leche, pastoreo.

\section{ABSTRAC}

The objective of the present work was to investigate if the practice of a dry-off period in goats maintained under grazing conditions, increases milk yield and therefore the quantity of its compounds. In goats without dry-off, females were hand milked until parturition (SPS; $n=9$ ). Hand milking of goats from dry-off period was stopped 50 days prior occurrence of parturition (CPS; $n=11$ ). We used a factorial design with a lineal model with fixed effects to compare milk yield and quantity of its main compounds, in response to the treatment, time, and time $\times$ treatment interaction. There was a treatment effect on milk yield during lactation, with higher values in CPS group than SPS group $(P<0.001)$. In the same way, the quantity of milk compounds was influenced

Volumen XXIII, Número 1 by the treatment $(P<0.001)$. In conclusion, the practice of the dry period in goats under grazing conditions, increase milk production during subsequent lactation and therefore the quantity of its components.

Keywords: Dry-off period, goats, lactation, milk yield, grazing.

\section{INTRODUCCIÓN}

De manera global, la mayoría de los sistemas de pastoreo extensivos caprinos están situados en zonas áridas o semiáridas (Albenzio et al., 2016). En México, existen tres regiones que practican la caprinocultura (noreste, centro y sureste) y esta actividad proporciona los ingresos a los productores en estas regiones (Montaldo et al., 2010). Sin embargo, muchas de las unidades de producción se caracterizan por tener un bajo nivel socioeconómico, escases de recursos naturales como el agua, y los animales son mantenidos en una vegetación nativa que presenta variaciones en cantidad y calidad nutricional a lo largo del año (Baraza et al., 2008). La Comarca Lagunera ubicada en la región noreste de México, posee un inventario poblacional de 416,820 caprinos y una producción de leche anual de 58,964,000 litros (SIAP, 2017).

En esta región de manera natural, las hembras adultas son preñadas en el mes de junio-julio, cuando los machos inician su periodo de actividad sexual. Una vez que las cabras son preñadas se desarrolla la gestación sin que el productor practique el periodo seco hacia el final de la gestación en las hembras y la mayoría de ellas presenten un secado natural paulatino (Escareño et al., 2011). Sin embargo, en rumiantes mantenidos en un sistema intensivo es bien conocido que cuando se practica el secado, ello resulta en una mayor producción de leche en la lactancia siguiente (Caja et al., 2006). El secado, es el periodo en el que las células alveolares dañadas o senescentes del tejido mamario son remplazadas para asegurar una producción de leche eficientemente en el periodo lactacional subsecuente (Capuco et al., 1997). Por ello, en cabras con un potencial lechero elevado y a las que se les practicó el periodo seco de 50 días produjeron más leche en la lactancia subsecuente que en las cabras que no se les aplicó el secado (Salama et al., 2005). Incuso, se ha demos-

*Autor para correspondencia: Horacio Hernández Hernández Correo electrónico: hernandezhoracio@outlook.com

Recibido: 30 de noviembre de 2019 Aceptado: 12 de marzo de 2020 
trado que en cabras lecheras mantenidas bajo un sistema semi-intensivo, que con solo 27 días de secado es suficiente para mejorar la calidad del calostro y leche (Caja et al., 2006). Hasta hoy, existe escasa información del efecto que tiene el practicar el periodo seco sobre la producción de leche en la lactancia subsecuente en animales mantenidos bajo condiciones de pastoreo. Por lo tanto, el objetivo de este estudio fue determinar si el practicar el periodo seco en cabras bajo condiciones de pastoreo incrementa la producción y por consecuencia la cantidad de los componentes químicos de la leche.

\section{MATERIALES Y MÉTODOS}

Los procedimientos experimentales reportados en el artículo actual estaban de acuerdo con la guía Investigación en Animales: Reportes de Experimentos en Vivo (ARRIVE; Kilkenny et al., 2010) y con la Norma Oficial Mexicana para las especificaciones técnicas para la producción, cuidado y uso de animales de laboratorio (NOM-062-ZOO-1999; Secretaría De Agricultura, Ganadería, Desarrollo Rural, Pesca y Alimentación (SAGARPA, 2001)).

\section{Localización del estudio}

El estudio se realizó en la parte subtropical norte de México, en la región de la Comarca Lagunera (Estado de Coahuila; latitud $26^{\circ} \mathrm{N} ; 1100 \mathrm{msnm}$ ). Las temperaturas máximas y mínimas promedio registradas durante el estudio fueron $24.6^{\circ} \mathrm{C}$ y $6.0^{\circ} \mathrm{C}$, respectivamente.

Animales, condiciones de manejo y diseño experimental

En el presente estudio se utilizaron 20 cabras locales multíparas encastadas (Capra hircus). Esta cabras se derivan de las razas española Granadina, Murciana y Malagueña, con cruzas de las razas Alpina, Saanen y Anglo-Nubian (Duarte et al., 2008). Todas las cabras se encontraban bajo un sistema de pastoreo extensivo. Éstas fueron pastoreadas diariamente de 10:00 a 13:00 horas y de 15:00 a 18:00 horas y permanecían en corrales durante la noche. Las cabras se alimentaron de la vegetación nativa y se complementaron con $0.2 \mathrm{~kg}$ de concentrado comercial el cual contenía 18\% de PC (Generaleche, Purina ${ }^{\oplus}$, Irapuato, México). Aproximadamente a 50 días previos a la fecha promedio del parto las cabras se asignaron de manera aleatoria a 1 de 2 grupos. Una vez conformados los grupos, no existió diferencia entre grupos en el número de fetos detectados por ultrasonografía transrectal, ni en la producción de leche previa, ni en la condición corporal. En el grupo de cabras sin periodo seco los animales se mantuvieron diariamente en ordeño manual hasta la ocurrencia del parto (SPS; $n=9$ ). En cambio, las cabras del grupo con periodo seco se practicó el secado al suspender el ordeño manual hasta el parto (CPS; $n=11$ ). En la última ordeña a estas cabras se les administró vía intramamaria $5 \mathrm{~mL}$ de un desecador comercial (Cepravin Intervet ${ }^{\circledR}$ Kenilworth, USA) para prevenir infecciones.

La fecha promedio \pm error estándar del promedio $( \pm$ SEM) del parto para todas las cabras fue el 31 de agosto
( \pm 1.0 día). Las crías se mantuvieron con su respectiva madre durante los primeros 21 días post-parto. Ellas fueron amamantadas libremente desde que sus madres regresaban del pastoreo hasta la mañana siguiente. Después del retiro definitivo de las crías, las cabras se ordeñaron una vez al día (a las 07:00 horas) hasta el final del experimento.

\section{Variables evaluadas}

Estimación de la producción de leche durante el preparto

Durante este periodo y solo en las cabras del grupo

SPS se midió de manera semanal la cantidad de leche obtenida en $24 \mathrm{~h}$. La leche obtenida en el ordeño se pesó en una báscula electrónica con una capacidad de $40 \mathrm{~kg}$ y una precisión de $0.005 \mathrm{~kg}$ y esta cantidad fue registrada en un formato.

\section{Estimación de la producción de leche durante el periodo de amamantamiento}

La producción de leche durante el periodo de amamantamiento para ambos grupos fue estimada por el método de la diferencia de peso de las crías antes y después de un amamantamiento controlado (Ricordeau et al., 1960). Éste consistió en el vaciado completo de la ubre a las 19:00 horas y la (s) cría (s) se separaron de su madre toda la noche. Al día siguiente a las 07:00 horas se pesó a la cría vacía, después de esto, se le permitió un amamantamiento controlado de $3 \mathrm{~min}$ y la (s) cría (s) nuevamente fueron pesadas. Por diferencia de pesos de la (s) cría (s) se obtuvo la producción láctea de la madre. Finalmente se administró $2 \mathrm{UI}$ de oxitocina exógena (OT; Oxilac-Proquivet, Guadalajara, México) para extraer la leche residual, la cual se sumó a la leche obtenida por la diferencia de pesos de la cría.

\section{Estimación de la producción de leche durante el periodo de ordeño}

Una vez que las crías fueron separadas definitivamente de sus madres, la producción de leche durante el periodo de ordeño fue evaluada cada semana durante los primeros 2.5 meses de lactación. Así, en cada ocasión se registró la cantidad de leche obtenida en la ordeña manual. Al final del ordeño, se administró por vía IV de la yugular 2 UI de OT exógena, e inmediatamente se procedió a ordeñar para extraer la leche residual, la cual se sumó a la leche obtenida en el ordeño.

\section{Cantidad obtenida de los componentes de la leche}

En cada ocasión cuando la producción de leche fue estimada, se tomó una muestra de leche de $20 \mathrm{~mL}$ de la ordeña manual la cual se recolectó en bolsas plásticas estériles etiquetadas, manteniéndolas en refrigeración y fueron transportadas a laboratorio. Utilizando un equipo automatizado (Milkoscope Expert ${ }^{\oplus}$, Scope Electric. Ratisbona, Alemania), se realizó el análisis de las muestras de leche y se obtuvieron los porcentajes de sólidos totales, grasa, lactosa y proteína. Para calcular en gramos/día los porcentajes de estos componentes se utilizó una regla de tres simple directa, considerando la producción de leche de cada cabra como el 100\%. 


\section{Condición corporal (CC)}

La CC se midió cada semana durante todo el estudio mediante la técnica descrita por Walkden-Brown et al. (1997), la cual consiste en estimar por palpación la cantidad de tejido muscular y graso de la región lumbar del animal. Esta metodología, utiliza una escala de valores que van del 1 a 4 puntos con una precisión de 0.5 .

\section{Mediciones de la ubre}

En las cabras de ambos grupos las mediciones externas de la ubre (vacía en el caso de las del grupo SPS) se registraron 1 semana antes de la fecha promedio esperada del parto. Se utilizó la técnica descrita por Emediato et al. (2008) para medir el ancho, profundidad y circunferencia de la ubre.

\section{Cálculo de variables y análisis estadístico}

Con los datos individuales de producción de leche de cada cabra obtenidos cada semana en ambos periodos (amamantamiento y ordeña) se calculó el promedio total durante el tiempo de estudio. Con cada uno de estos datos se calculó el promedio ponderado de cada grupo y este se comparó con una $t$ de student independiente. Se utilizó un diseño factorial con un modelo lineal de efectos fijos para evaluar la producción y los compuestos químicos de la leche en respuesta al factor tratamiento (dos niveles: SPS y CPS), el factor tiempo (14 niveles, correspondientes a las semanas de medición durante la segunda lactancia del experimento) y la interacción tiempoxtratamiento en el periodo amamantamiento y ordeña.

El modelo usado fue:

$Y_{i j k}=\mu+$ tratamiento $_{i}+$ tiempo $_{j}+$ tratamientoxtiempo $_{i j}$ $+\mathrm{e}_{\mathrm{ij} \mathrm{k}^{\prime}}$ donde:

$Y_{i j k}$ es el ijkiésima observación de la variable medida (producción de leche, y componentes químicos), $\mu$ es la media general, tratamiento es el efecto del iésimo tratamiento, tiempo $o_{j}$ es el efecto de la jiésima semana de medición, tratamientoxtiempo $\mathrm{i}_{\mathrm{ij}}$ es la interacción entre tratamiento $y$ tiempo, y $e_{i j \mathrm{j}}$ representa el error aleatorio asociado a cada observación.

Los datos obtenidos de cada medición que fueron utilizados para cada variable como las mediciones de la ubre, el peso de las crías y su tasa de crecimiento se compararon entre grupos con una $t$ de student independiente. Todos los análisis se llevaron a cabo utilizando el paquete estadístico Statgraphics plus versión (Statpoint, Herndon, VA). El nivel de significancia se estableció a $P<0.05$.

\section{RESULTADOS Y DISCUSIÓN}

En la Figura 1, se observa que la producción de leche varió a través del tiempo para ambos grupos $(P<0.001)$. Existió un efecto del tratamiento sobre la producción de leche durante el periodo de amamantamiento y de ordeño, por lo que ésta fue mayor para el grupo CPS que para el

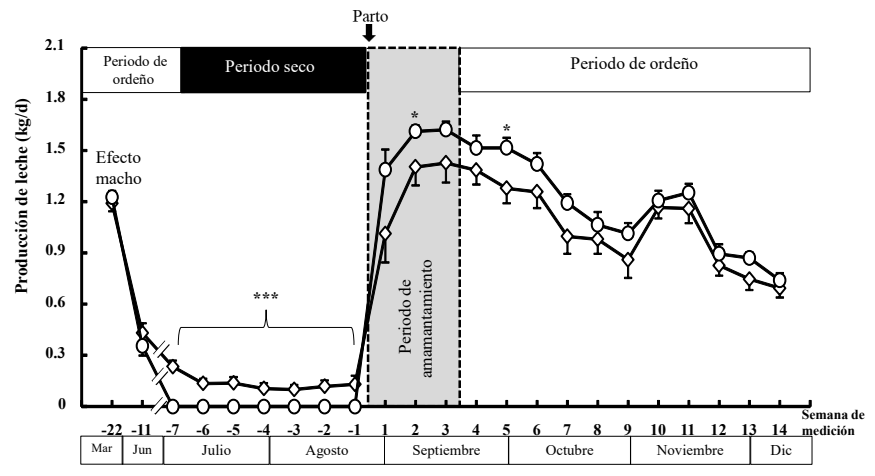

Figure 1: Least squares means $( \pm S E)$ of milk production of the grazing goats group that underwent dry period (DP group; $\circ ; n=11$ ), and from the group without dry period (NDP group; $\diamond ; n=9$ ). Significant differences between treatments are indicated by asterisks $\left({ }^{*} P<0.05\right.$ and $\left.{ }^{* * *} P<0.001\right)$. Figura 1. Medias de mínimos cuadrados ( \pm EE) de producción de leche del grupo de cabras en pastoreo a las que se les practicó periodo seco (grupo CPS; $\circ ; n=11$ ), y del grupo que no se practicó el periodo seco (grupo SPS; $\diamond ; \mathrm{n}=9$ ). Diferencias significativas entre tratamientos en cada semana son indicados por los asteriscos $\left({ }^{*} P<0.05 y{ }^{* * *} P<0.001\right)$.

Tabla 1. Valores medios $( \pm E E)$ de variables de producción, dimensiones de la ubre y parámetros de crías de las cabras del grupo con período seco (CPS, $\mathrm{n}=$ 11), y sin período seco (SPS, $n=9$ ).

Table 1. Means $( \pm S E)$ of production variables, udder dimensions and calf parameters of dry period group goats (DP, $n=11)$ and without dry period (NDP, $n=9$ ).

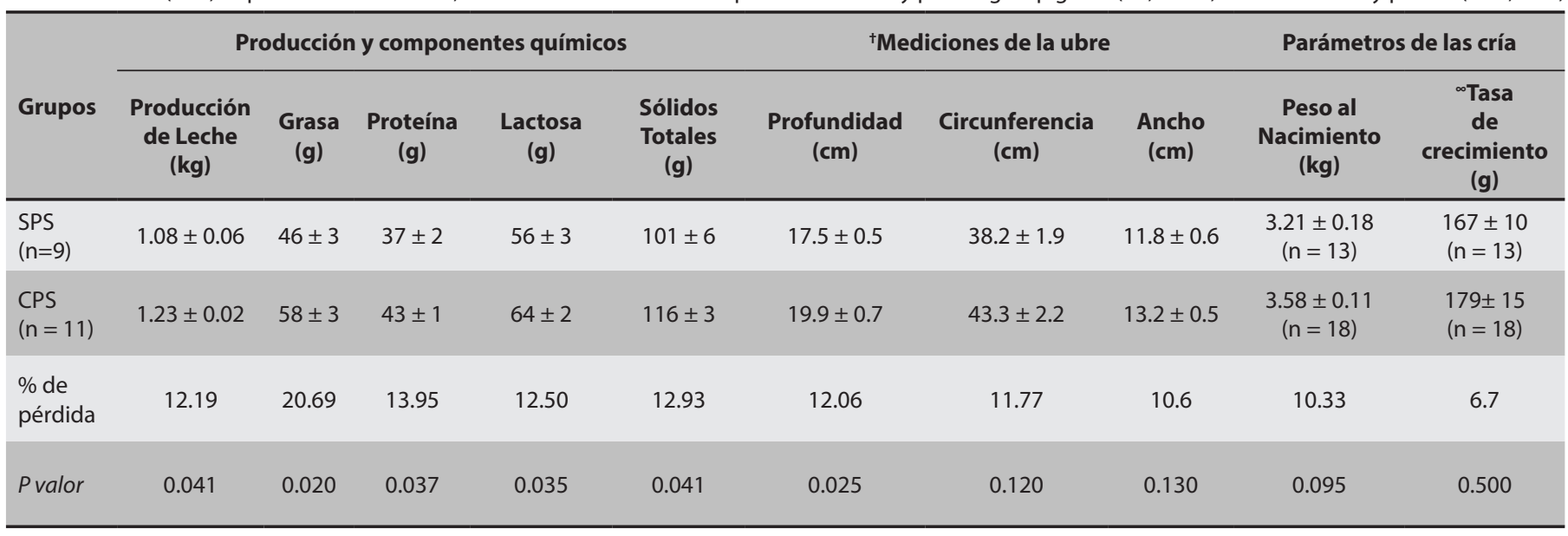

Nota: ${ }^{\dagger}$ Las mediciones de ubre se llevaron a cabo una semana previa a la fecha promedio del parto.

${ }^{\infty}$ La tasa de crecimiento de las crías de calculó a los 21 días de vida. 
grupo SPS $(\mathrm{P}<0.001)$. Los análisis estadísticos demostraron que la interacción tratamiento $\times$ tiempo no fue significativa $(P>0.05)$. Los resultados presentados en la Tabla 1, refuerzan lo anterior, ya que se observan hasta el periodo evaluado porcentajes de pérdidas tanto para la producción como para sus componentes químicos. Los presentes resultados confirman que en cabras mantenidas en pastoreo extensivo el inducir un periodo de descanso de la ubre o periodo seco repercute de manera importante sobre la producción de leche en la lactancia subsecuente. Estos resultados concuerdan con los obtenidos por Caja et al. (2006) quienes observaron que en las cabras con alto potencial lechero mantenidas en un sistema semi-intensivo y a las que se les omitió practicar un periodo de secado produjeron menos leche que a las que se les practicó dicho periodo seco. Además, este mismo equipo de investigadores demostró que en la cabra MurcianoGranadina que de manera espontánea se secaron por solo 27 días, su producción de leche subsecuente no difirió de aquellas que fueron secadas por 50 días.

La producción por día de los diferentes contenidos evaluados en la leche mostró variaciones a través del tiempo $(P<0.001)$, como se muestra en la Figura 2. En ella, además se observa que la cantidad por día en todos los componentes fue mayor en las cabras del grupo CPS que en las del grupo SPS, sobre todo en el periodo que permanecieron las crías con sus madres. En efecto, el modelo estadístico indicó en los cuatro componentes un efecto significativo del tratamiento $(P<0.001)$. Estos resultados aportan información novedosa en los rumiantes en general. Efectivamente, no se tiene literatura acerca de cómo los componentes químicos de la leche se disminuyen por efecto de la práctica de no del secado, sobre todo en la lactancia temprana. Entre los pocos estudios en cabras, Caja et al. (2006), observaron que los componentes del calostro disminuyen por efecto de la práctica del no secado, que en aquellas que se sometieron al secado. En las vacas Holstein italianas, Mantovani et al. (2010) no encontró diferencia en el porcentaje de grasa y proteína contenida en la leche de vacas que fueron secadas por 50 días y de aquellas que no fueron secadas. Sin embargo, al igual que en el presente estudio, como la producción de leche fue mayor en las vacas que fueron secadas, ello resultaría en mayor producción en gramos/día de esos componentes de la leche. Sin embargo, estudios en los cuales se establecieron diferentes duraciones del periodo seco en vacas, indicaron que a menor tiempo de secado se obtienen menores cantidades de los componentes de la leche que a mayor tiempo de secado (Sorensen y Enevoldsen, 1991).

Las mediciones de la profundidad, la circunferencia y el ancho de la ubre evaluadas a una semana antes del parto se muestran en la Tabla 1. En esta tabla es claro el efecto que ejerce el ordeño continuo (grupo SPS) sobre esas mediciones, ya que todas fueron mayores en el grupo CPS; sin embargo, solo la profundidad de la ubre alcanzó significancia $(P<0.05)$. Posiblemente este crecimiento de la ubre hacia el final del periodo seco en las cabras del grupo CPS es reflejo de un proceso de hipertrofia en el parénquima mamario (Turner, 1965).
La tasa de crecimiento de las crías del grupo CPS fue mayor que el registrado en las crías del grupo SPS, aunque no hubo diferencia significativa ( $P>0.05$; Tabla 1$)$. Es probable que como se ha reportado previamente (García y González et al., 2017) el peso de las crías está correlacionado positivamente con el nivel de producción láctea de las madres. En otras palabras, es posible que como las madres del grupo CPS produjeron mayor cantidad de leche entonces sus crías fueron más pesadas que en las del grupo SPS.

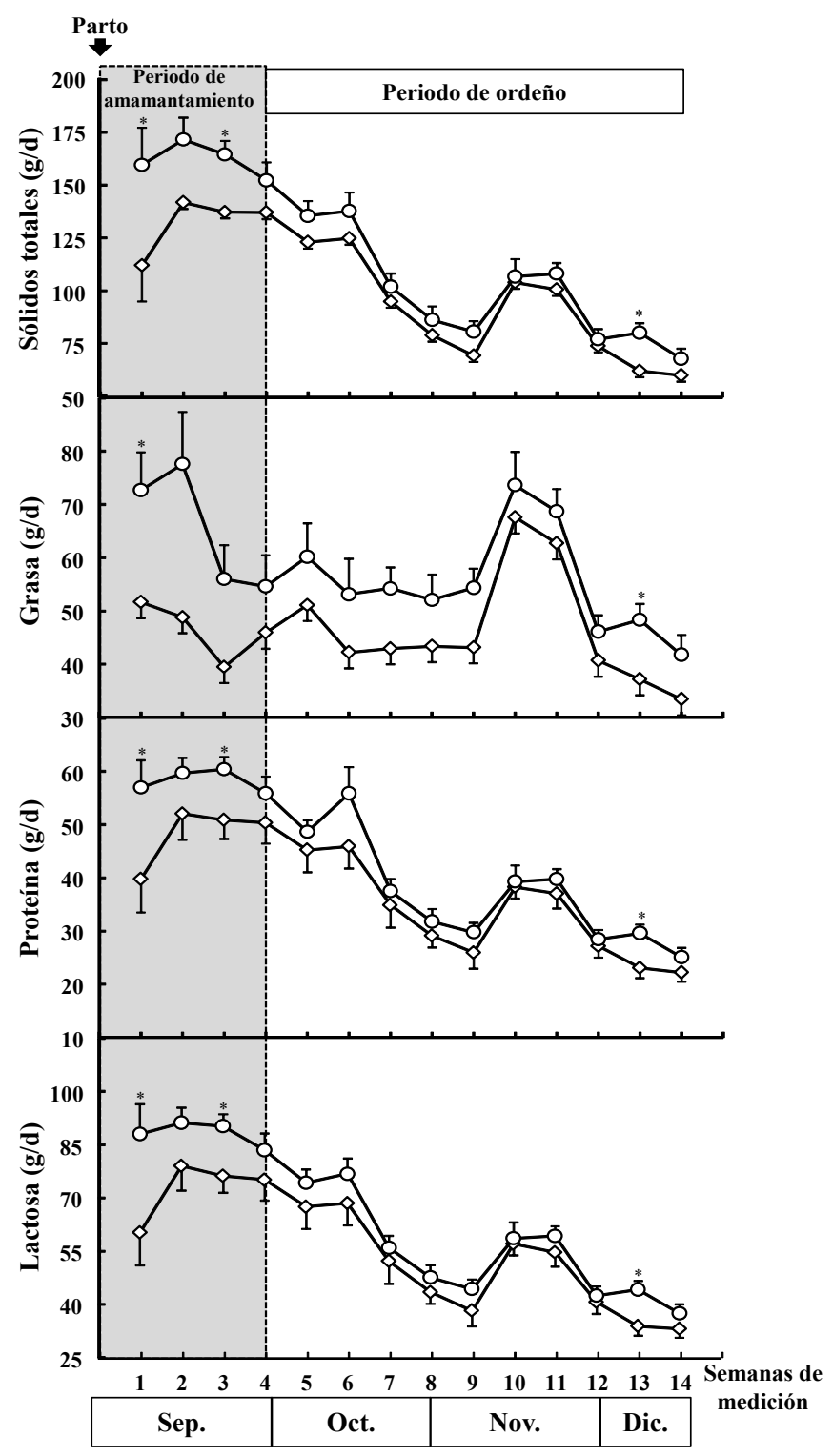

Figure 2. Least squares means ( $\pm \mathrm{SE}$ ) of total solids, fat, protein and lactose content of the grazing goats group that underwent dry period (DP group; $\circ ; \mathrm{n}=11$ ), and from the group without dry period group (NDP group; $0 ; \mathrm{n}$ $=9$ ). Significant differences between treatments are indicated by asterisks $\left({ }^{*} \mathrm{P}<0.05\right)$.

Figura 2. Medias de mínimos cuadrados ( $\pm \mathrm{EE}$ ) del contenido de sólidos totales, grasa, proteína y lactosa de cabras en pastoreo a las que se les practicó periodo seco (grupo CPS; $;$, $=11$ ), y cabras sin período seco (grupo SPS; $\diamond ; n=9$ ). Diferencias significativas entre tratamientos en cada semana son indicados por asteriscos ( $\left.{ }^{*} \mathrm{P}<0.05\right)$. 
La CC en las cabras de ambos grupos en diferentes periodos del estudio se muestra en la Figura 3. No se encontró un efecto del tratamiento $(\mathrm{P}>0.05)$ sobre esta variable en ningún momento del estudio. Sin embargo, en ambos grupos esta variable mostró variaciones a través del tiempo $(P<0.001)$. Aunque en el presente estudio la $C C$ no fue afectada por el tratamiento, en vacas lecheras se ha reportado que las hembras que ganan CC durante el periodo seco producen más leche, grasa y proteína que aquellas que no ganan CC (Chebel et al., 2018).

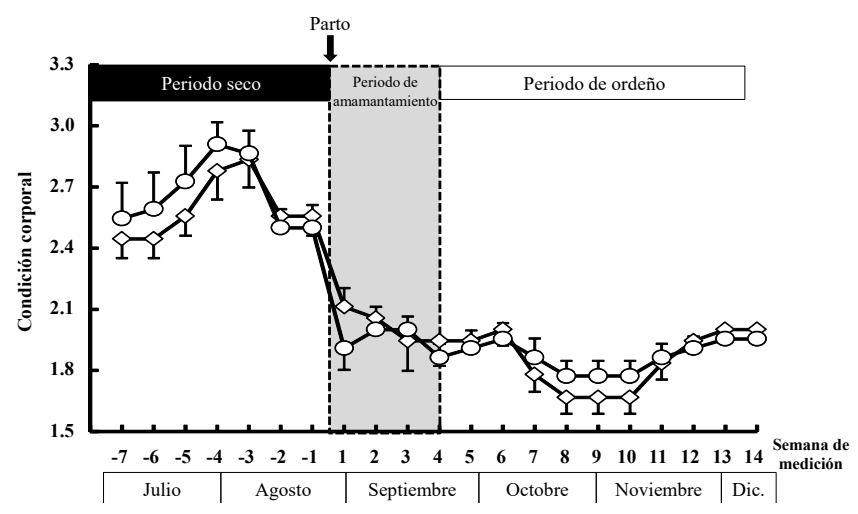

Figure 3: Least squares means $( \pm S E)$ of body condition score of the grazing goats group that underwent dry period (DP group; $\circ ; n=11$ ), and from the group without dry period (NDP group; $\diamond ; n=9$ ).

Figura 3. Medias de mínimos cuadrados $( \pm S E M)$ de la condición corporal de cabras en pastoreo a las que se les practicó periodo seco (grupo CPS; $\circ$; $n=11$ ), y cabras sin período seco (grupo SPS; $\diamond ; n=9$ ).

\section{CONCLUSIONES}

Los resultados obtenidos en el presente estudio nos permiten concluir que, en las cabras mantenidas bajo un sistema extensivo, el practicar un periodo de secado de 50 días promueve de manera importante una mayor producción láctea tanto en el periodo de amamantamiento como durante el periodo de ordeño. Debido a este incremento, también se obtiene una mayor cantidad diaria de los principales componentes de la leche. Sería importante determinar, como se ha hecho en cabras con mayor potencial lechero, si este periodo de secado pueda reducirse, sin comprometer el desempeño de la lactancia subsecuente.

\section{AGRADECIMIENTOS}

Los autores agradecen al Prof. Jesús Abasta por la facilitación de los animales utilizados en el presente estudio. Asimismo, agradecen a la Lic. Dolores López Magaña, a Esther Peña y a todos los Investigadores y alumnos del CIRCA-UAAAN y del Posgrado en Ciencias Agrarias por su apoyo técnico para la realización del presente estudio.

R. Avilés fue apoyado por una beca CONACyT durante sus estudios de Doctorado. La investigación fue realizada como parte de las actividades del Laboratorio Internacional Asociado (LIA CABRAA) entre México (UAAAN-CIRCA) y Francia (INRA-PRC).

\section{REFERENCIAS}

Albenzio, M., Santillo, A., Avondo, M., Nudda, A., Chessa, S., Pirisi, A. and Banni, S. 2016. Nutritional properties of small ruminant food products and their role in human health. Small Ruminant Research. 135:3-12.

Baraza, E., Ángeles, S., García, A. y Valiente, B.A. 2008. Nuevos recursos naturales como complemento de la dieta de caprinos durante la época seca, en el Valle de Tehuacán, México. Interciencia. 33:12-15.

Caja, G., Salama, A.A.K. and Such, X. 2006. Omitting the dry-off period negatively affects colostrum and milk yield in dairy goats. Journal of Dairy Science. 89:4220-4228.

Capuco, A.V., Akers, R.M. and Smith, J.J. 1997. Mammary growth in Holstein cows during the dry period: Quantification of nucleic acids and histology. Journal of Dairy Science. 80:477487.

Chebel, R.C., Mendonça, L.G.D. and Baruselli, P.S. 2018. Association between body condition score change during the dry period and postpartum health and performance. Journal of Dairy Science. 101:4595-4614.

Duarte, G., Flores, J.A., Malpaux, B. and Delgadillo, J.A. 2008. Reproductive seasonality in female goats adapted to a subtropical environment persists independently of food availability. Domestic Animal Endocrinology. 35:362-370.

Emediato, R.M.R., Siqueira, E.R., Stradiotto, M.M., Maesta, S.A. and Fernández, S. 2008. Relationship between udder measurements and milk yield in Bergamasca in Brazil. Small Ruminant Research. 75:232-235.

Escareño, L., Wurzinger, M., Pastor, F., Salinas, H., Solkner, J. y Iñiguez, L. 2011. La cabra y los sistemas de producción caprina de los pequeños productores de la Comarca Lagunera, en el norte de México. Revista Chapingo Serie Ciencias Forestales y del Ambiente. 17:235-246.

García y González, E., Flores, J.A., Delgadillo, J.A., GonzálezQuirino, T., Fernández, I.G., Terrazas, A. and Hernández, H. 2017. Early nursing behaviour in ungulate mothers with hider offspring (Capra hircus): Correlations between milk yield and kid weight. Small Ruminant Research. 151:59-65.

Kilkenny, C., Browne, W.J., Cuthill, I.C., Emerson, M. and Altman, D.G. 2010. Animal research: reporting in vivo. Public Library of Science. Biology. 8, e1000412.

Mantovani, R., Marinelli, L., Bailoni, L., Gabai, G. and Bittante G. 2010. Omission of dry period and effects on the subsequent lactation curve and on milk quality around calving in Italian Holstein cows. Italian Journal of Animal Science. 9:110-118.

Montaldo, H.H., Torres-Hernández, G. and Valencia-Posadas, M. 2010. Goat breeding research in México. Small Ruminant Research. 89:155-163.

Ricordeau, G., Boccard, R. and Denamur, R. 1960. Mesure de la production laiti冈re des brebis pendant la période d'allaitement. Annales de Zootechnie. 9:97-120.

SAGARPA. 2001. Norma Oficial Mexicana NOM-062-ZOO-1999, Especificaciones técnicas para la producción, cuidado y uso de los animales de laboratorio. Diario Oficial de la Federación. June $18^{\text {th }}, 2001$. Ciudad de México, México.

Salama, A.A.L., Caja, G., Such, X., Cassals, R. and Albanell, E. 2005. Effect of pregnancy and extended lactation on milk production in dairy goats milked once daily. Journal of Dairy Science. 88:3894-3904.

SIAP. 2017: Población caprina en México 2008-2017. Secretaría de agricultura, ganadería desarrollo rural pesca y alimentación. 
Ciudad de México, México.http://www.siap.gob.mx/opt/ poblagand/caprino.pdf (12/05/2017).

SØrensen, J.T. and Enevoldsen, C. 1991. Effect of dry period length on milk production in subsequent lactation. Journal of Dairy Science. 79:1277-1283.

Turner, C.W. 1965. What cause high production? Boletín 836. University of Missouri. Agricultural Experiment Station.
Walkden-Brown, S.W., Restall, B.J., Scaramuzzi, R.J, Martin, G.B. and Blackberry, M.A. 1997. Seasonality in male Australian cashmere goats: long term effects of castration and testosterone or oestradiol treatment on changes in $\mathrm{LH}$, $\mathrm{FSH}$, and prolactin concentrations, and body growth. Small Ruminant Research. 26:239-252. 